\title{
SHAKEDOWN ANALYSIS OF PLATE BENDING UNDER STOCHASTIC UNCERTAINITY BY CHANCE CONSTRAINT PROGRAMMING
}

\author{
Ngọc Trình Trần ${ }^{1}$, Thanh Ngọc Trần ${ }^{2}$, H.G. Matthies ${ }^{3}$, G.E. Stavroulakis ${ }^{4}$ and M. Staat ${ }^{1}$ \\ ${ }^{1}$ Aachen University of Applied Sciences, Jülich Campus, Institute for Bioengineering, Biomechanics \\ Lab., Heinrich-Mußmann-Str. 1, 52428 Jülich, Germany, trinhdhkt@gmail.com, m.staat@fh- \\ aachen.de, http://www.fh-aachen.de/forschung/institut-fuer-bioengineering/ \\ ${ }^{2}$ University of Duisburg-Essen, Chair of Mechanics and Robotics, Lotharstr. 1, 47057 Duisburg, \\ Germany, thanh.tran@uni-due.de \\ ${ }^{3}$ TU Braunschweig, Institute of Scientific Computing, Hans-Sommer-Str. 65, 38092 Braunschweig, \\ Germany, wire@tu-bs.de \\ ${ }^{4}$ Technical University of Crete, 73100 Chania, Greece, gestavr@dpem.tuc.gr
}

Keywords: Limit analysis, Shakedown analysis, stochastic programming, chance constrained programming, non-linear programming.

\begin{abstract}
In this paper we propose a stochastic programming to analyze limit and shakedown of plate bending under uncertainty conditions of strength. The Kirchhoff plate theory is used to formulate chance constrained problems. Based on the duality theory, the shakedown load multiplier formulated by the kinematic theorem is proved actually to be the dual form of the shakedown load multiplier formulated by static theorem. In this investigation a dual chance constrained programming algorithm is developed to calculate simultaneously both the upper and lower bounds of the plastic collapse limit and the shakedown limit.
\end{abstract}




\section{INTRODUCTION}

An essential concern in mechanical and civil engineering design is to determine the ultimate load bearing capacity of structures beyond the elastic limit. Structures are considered safe if a state is reached after initial plastic deformation, such that the system does neither fail due to instantaneous or incremental collapse nor due to alternating plasticity. Using the classical step-by-step methods for such calculations can be cumbersome and computationally expensive in many cases. Furthermore, the disadvantage of these methods is that the exact knowledge of the loading history is necessary, which is not realistic in many technical applications. Limit and shakedown analysis are appropriate direct methods to avoid these problems. Reliability analysis of the structures takes the uncertainty of the actual load-carrying capacity of the structure into consideration since all resistance and loading variables are random in nature $[1,9,10,20]$.

Chance constrained programing is an approach of stochastic programming to limit and shakedown analysis under uncertainty $[1,2]$. Under uncertainty the shakedown problem can be stated with random objective function or with random constraints. A probability is set with which the constraint has to be satisfied. Deterministic limit analysis of plates subjected to bending has been studied analytically and numerically. Both the Kirchhoff plate and the Mindlin plate models are considered based on the kinematic theorem of limit analysis and the finite element method. Based on the duality theory, Tran [3] developed a dual algorithm to calculate simultaneously both the upper and lower bounds of the plastic collapse limit and shakedown load of plates. In this study, we present a new primal-dual numerical algorithm of the shakedown problem under uncertainty in which the material strength is considered as a normally distributed random variable. Using chance constrained programming, we obtain deterministic equivalent formulations based on upper bound and lower bound theorems and then prove that formulations are actually dual to each other. The four-node discrete Kirchhoff quadrilateral (DKQ) element is used to analyze the problem. The proposed algorithm shows good performance in numerical test examples.

\section{SHAKEDOWN ANALYSIS OF PLATE BENDING}

\subsection{Basic relations}

Similar to the elastic theory, the inelastic behavior of thin plates may be analyzed under Kirchhoff's assumption that the normal to the middle plane of the plate remains straight and normal to the deformed middle plane. This assumption yields $u=-z \frac{\partial w}{\partial x}, v=-z \frac{\partial w}{\partial y}$, in which $x, y$ are the coordinates in the middle plane of the plate, $z$ is the distance from the middle plane, $w$ is the deflection, $u$ and $v$ are the displacements in the $x$ and $y$ directions respectively. By differentiation, the strains are obtained as

$$
\left\{\begin{array}{c}
\varepsilon_{x x} \\
\varepsilon_{y y} \\
\gamma_{x y}
\end{array}\right\}=\left\{\begin{array}{c}
\frac{\partial u}{\partial x} \\
\frac{\partial v}{\partial y} \\
\frac{\partial v}{\partial x}+\frac{\partial u}{\partial y}
\end{array}\right\}=\left\{\begin{array}{c}
-z \frac{\partial^{2} w}{\partial x^{2}} \\
-z \frac{\partial^{2} w}{\partial y^{2}} \\
-2 z \frac{\partial^{2} w}{\partial x \partial y}
\end{array}\right\}=z\left\{\begin{array}{c}
\chi_{x} \\
\chi_{y} \\
\chi_{x y}
\end{array}\right\}
$$


The vector $\chi=\left[\begin{array}{lll}\chi_{x} & \chi_{y} & \chi_{x y}\end{array}\right]^{T}$ is called the vector of curvatures.

The kinematic relations can be written as follows:

$$
\dot{\chi}=-\nabla^{2} \dot{w}
$$

where $\dot{\chi}$ is the curvature rate vector and $\dot{w}$ is the transversal velocity.

In this paper, the von Mises yield criterion is considered. Expressing in terms of moments, the criterion takes the form $f\left(m_{x}, m_{y}, m_{x y}\right)=\left(m_{x}^{2}-m_{x} m_{y}+m_{y}^{2}+3 m_{x y}^{2}\right)^{1 / 2}-m_{0}=0$.

In matrix form the von Mises yield criterion can be written as follows:

$$
f(\mathbf{m})=\sqrt{\mathbf{m}^{\mathrm{T}} \mathbf{P m}}-m_{0}=0
$$

where $\mathbf{m}=\left[m_{x}, m_{y}, m_{x y}\right]^{\mathrm{T}}$ is the vector of bending and twisting moments, $m_{0}=\sigma_{y} t^{2} / 4$ is the plastic limit moment per unit length of a plate section, $t$ is the thickness of the plate, $\sigma_{y}$ is the yield stress of the material, and

$$
\mathbf{P}=\frac{1}{2}\left[\begin{array}{lrr}
2 & -1 & 0 \\
-1 & 2 & 0 \\
0 & 0 & 6
\end{array}\right]
$$

\subsection{Static formulation}

Consider a convex polyhedral load domain $\mathcal{L}$ and a special loading path consisting of all load vertices $\hat{P}_{k}(k=1, \ldots, m) \in \mathcal{L}$. Let a point on the problem domain $A$ (the midplane) be identified by a vector variable $\mathbf{x}$ and the fictitious elastic moment vector be $\mathbf{m}^{\mathrm{E}}$. The static shakedown theorem states that shakedown occurs if, and only if, there exists a timeindependent self-equilibrium residual moment field $\overline{\boldsymbol{\rho}}$ which is statically admisible so that the actual moment field $\mathbf{m}=\alpha \mathbf{m}^{\mathrm{E}}+\overline{\boldsymbol{\rho}}$ does not anywhere violate the yield condition at any point of the structure and for all posible load combinations. Based on this theorem, we can find a statically admisible residual moment field in order to obtain a maximum load domain $\alpha \mathcal{L}$ that guarantees (3). Therefore, the shakedown load factor $\alpha^{-}$can be obtained by solving the following optimization problem:

$$
\begin{array}{r}
\alpha^{-}=\max \alpha \\
\text { s.t. }:\left\{\begin{array}{l}
\nabla^{2} \overline{\boldsymbol{\rho}}(\mathbf{x})=0 \quad \text { in } A \\
f\left(\alpha \mathbf{m}^{\mathrm{E}}+\overline{\boldsymbol{\rho}}\right) \leq m_{0}
\end{array}\right.
\end{array}
$$

By discretizing the entire problem domain into finite elements and applying the GaussLegendre integration technique, Eqs (5) can be rewritten in the following form: 


$$
\begin{aligned}
& \alpha^{-}=\max \alpha \\
& \text { s.t.: }\left\{\begin{array}{l}
\sum_{i=1}^{N G} w_{i} \mathbf{B}_{i}^{T} \overline{\mathbf{p}}_{i}=0 \quad \text { in } A \\
f\left(\alpha \mathbf{m}_{i k}^{E}+\overline{\boldsymbol{\rho}}_{i}\right) \leq m_{0} \quad \forall i=\overline{1, N G}, \quad \forall k=\overline{1, m}
\end{array}\right.
\end{aligned}
$$

in which $\mathbf{B}_{i}$ is the deformation matrix, $w_{i}$ is integration weight at Gauss point $i$; $N G$ denotes the total number of Gauss points of the structure, and $m$ is the number of load vetices..

\subsection{Kinematic formulation}

An upper bound to the shakedown limit of plate can be obtained using the kinematic shakedown theorem which has the following two statements:

(a) Shakedown will occur for a structure subject to repeated or cyclic loads, if the plastic dissipation power exceeds the work rate of external forces for any admissible plastic strainrate cycles and all loading paths

(b) Shakedown cannot occur, if the plastic dissipation power is less than the work rate of external forces for any one admissible plastic strain-rate cycle or any one loading path.

We introduce here an admissible cycle of plastic curvature field $\Delta \chi^{p}$. At each load vertex, the plastic curvature rate may not necessarily be compatible at each instant during the time cycle, but the plastic curvature accumulation over the cycle is required to be kinematically compatible such that

$$
\Delta \chi^{p}=\sum_{k=1}^{m} \dot{\chi}_{k}^{p}=\nabla^{2} \dot{w}
$$

Based on the above statements and the mathematical programming theory, an upper bound of the shakedown load factor can be found by solving the following convex nonlinear programming (the superscript $p$ is neglected for simplicity):

$$
\begin{aligned}
\alpha^{+}=\min & \sum_{k=1}^{m} \int_{A} D_{\text {int }}\left(\dot{\chi}_{k}\right) d A \\
\text { s.t.: } & \left\{\begin{array}{l}
\Delta \chi=\sum_{k=1}^{m} \dot{\chi}_{k}=\nabla^{2} \dot{w} \quad \text { in } A \\
\dot{w}=0 \quad \text { on } \partial A \\
\sum_{k=1}^{m} \int_{A} \mathbf{m}^{E}\left(x, \hat{P}_{k}\right)^{T} \dot{\chi}_{k} d A=1
\end{array}\right.
\end{aligned}
$$

where $D_{\text {int }}(\chi)$ is the plastic dissipation power per unit area. According to the von Mises yield condition, the plastic dissipation can be expressed in term of the curvature rates of the middle surface: $D_{\text {int }}(\dot{\chi})=m_{0} \sqrt{\dot{\boldsymbol{\chi}}_{k}^{T} \mathbf{Q} \dot{\chi}_{k}}$

with 


$$
\mathbf{Q}=\mathbf{P}^{-1}=\frac{1}{3}\left[\begin{array}{lll}
4 & 2 & 0 \\
2 & 4 & 0 \\
0 & 0 & 1
\end{array}\right]
$$

We denote the nodal variables of the finite element by $\mathbf{q}=\left[\begin{array}{lll}w & \partial w / \partial x & \partial w / \partial y\end{array}\right]^{\mathrm{T}}$, the discretized formulation by FEM is as follows:

$$
\begin{aligned}
& \alpha^{+}=\min \sum_{k=1}^{m} \sum_{i=1}^{N G} w_{i} m_{0} \sqrt{\dot{\boldsymbol{\chi}}_{i k}^{T} \mathbf{Q} \dot{\boldsymbol{\chi}}_{i k}} \\
& \text { s.t.: }\left\{\begin{array}{l}
\sum_{k=1}^{m} \dot{\boldsymbol{\chi}}_{i k}=\mathbf{B}_{i} \dot{\mathbf{q}} \quad \forall i=\overline{1, N G} \\
\sum_{k=1}^{m} \sum_{i=1}^{N G} w_{i} \dot{\boldsymbol{\chi}}_{i k}^{T} \mathbf{m}_{i k}^{E}=1
\end{array}\right.
\end{aligned}
$$

\section{STOCHASTIC SHAKEDOWN ANALYSIS OF PLATE BENDING BY CHANCE CONSTRAINED PROGRAMMING}

\subsection{Static Approach to chance constrained programming}

Consider the situation that the strength of material is not given, fixed quantity, but must be modelled $R=R(\omega)$ through random variables on a certain probability space. Hence, the shakedown problem (6),(10), respectively, under stochastic uncertainty must be reformulated by appropriate deterministic problems which are provided by adopting stochastic optimization techniques. A technique used effectively called chance constrained programming is presented here.

For stochastic plastic moment $\mathbf{m}_{i}(\omega)$ depending on some random $\omega$, a stochastic formulation can be obtained by assuming that the inequality constraints of (6) are satisfied at least by a chance $\psi=[0.5 ; 1]$ (or reliability level) at Gauss point $i$.

$\alpha^{-}=\max \alpha$

$$
\text { s.t.: }\left\{\begin{array}{l}
\sum_{i=1}^{N G} w_{i} \mathbf{B}_{i}^{T} \overline{\mathbf{\rho}}_{i}=0 \quad \text { in } A \\
\operatorname{Prob}\left[f\left(\alpha \mathbf{m}_{i k}^{E}+\overline{\boldsymbol{\rho}}_{i}\right)-m_{0 i}(\omega) \leq 0\right] \geq \psi \quad \forall i=\overline{1, N G}, \quad \forall k=\overline{1, m}
\end{array}\right.
$$

Based on the methodology of chance constrained programming, the stochastic program (11) is to be relaxed into an equivalent deterministic optimization problem:

$$
\begin{aligned}
& \alpha^{-}=\max \alpha \\
& \text { s.t.: }\left\{\begin{array}{l}
\sum_{i=1}^{N G} w_{i} \mathbf{B}_{i}^{T} \overline{\mathbf{\rho}}_{i}=0 \quad \text { in } A \\
f\left(\alpha \mathbf{m}_{i k}^{E}+\overline{\boldsymbol{\rho}}_{i}\right) \leq \mu_{i}-\kappa_{i} \sigma_{i} \quad \forall i=\overline{1, N G,} \quad \forall k=\overline{1, m}
\end{array}\right.
\end{aligned}
$$


where $\mu_{i}, \sigma_{i}, \kappa_{i}=\Phi^{-1}\left(\psi_{i}\right)$ is the mean value, standard deviation and the inverse of the cumulative distribution function of plastic moment at Gauss point $i$, respectively.

\subsection{Kinematic approach to chance constrained programming}

As mentioned above the deterministic formulation to calculate an upper bound shakedown load factor:

$$
\begin{aligned}
& \alpha^{+}=\min \sum_{k=1}^{m} \sum_{i=1}^{N G} w_{i} m_{0} \sqrt{\dot{\boldsymbol{\chi}}_{i k}^{T} \mathbf{Q} \dot{\boldsymbol{\chi}}_{i k}} \\
& \text { s.t.: }\left\{\begin{array}{l}
\sum_{k=1}^{m} \dot{\boldsymbol{\chi}}_{i k}=\mathbf{B}_{i} \dot{\mathbf{q}} \quad \forall i=\overline{1, N G} \\
\sum_{k=1}^{m} \sum_{i=1}^{N G} w_{i} \dot{\boldsymbol{\chi}}_{i k}^{T} \mathbf{m}_{i k}^{E}=1
\end{array}\right.
\end{aligned}
$$

If the yield stress of material is random, then the plastic moment is uncertainty quantity and the objective function of (13) is a stochastic variable. Firstly, we must properly define the minimum of a random function. This can be done in such a way that one looks for a minimum lower bound $\eta$ objective function under the constraint that the probability of violation of that bound is prescribed [4].

$\min \eta$

$$
\text { s.t.: }\left\{\begin{array}{l}
\operatorname{Prob}\left(\sum_{k=1}^{m} \sum_{i=1}^{N G} w_{i} m_{0}(\omega) \sqrt{\dot{\boldsymbol{\chi}}_{i k}^{\mathrm{T}} \mathbf{Q} \dot{\boldsymbol{\chi}}_{i k}} \geq \eta\right)=\psi \\
\sum_{k=1}^{m} \dot{\boldsymbol{\chi}}_{i k}=\mathbf{B}_{i} \dot{\mathbf{q}} \quad \forall i=\overline{1, N G} \\
\sum_{k=1}^{m} \sum_{i=1}^{N G} w_{i} \dot{\boldsymbol{\chi}}_{i k}^{\mathrm{T}} \mathbf{m}_{i k}^{E}=1
\end{array}\right.
$$

The chance constrained program technique also can convert the stochastic program (14) into an equivalent deterministic program as follows:

$$
\begin{aligned}
& \alpha^{+}=\min \sum_{k=1}^{m} \sum_{i=1}^{N G} w_{i}\left(\mu_{i}-\kappa_{i} \sigma_{i}\right) \sqrt{\dot{\chi}_{i k}^{\mathrm{T}} \mathbf{Q} \dot{\chi}_{i k}} \\
& \text { s.t.: }\left\{\begin{array}{l}
\sum_{k=1}^{m} \dot{\chi}_{i k}=\mathbf{B}_{i} \dot{\mathbf{q}} \quad \forall i=\overline{1, N G} \\
\sum_{k=1}^{m} \sum_{i=1}^{N G} w_{i} \dot{\boldsymbol{\chi}}_{i k}^{\mathrm{T}} \mathbf{m}_{i k}^{E}=1
\end{array}\right.
\end{aligned}
$$

\section{DUALITY APPROACH TO CHANCE CONSTRAINED PROGRAMMING}

\subsection{Dual theorem for chance constrained programming}

Some new notations is intruduced for the sake of simplicity: 


$$
\dot{\mathbf{k}}_{i k}=w_{i} \mathbf{Q}^{1 / 2} \dot{\chi}_{i k}, \quad \mathbf{t}_{i k}=\left(\mathbf{Q}^{-1 / 2}\right)^{T} \mathbf{m}_{i k}^{E}, \quad \hat{\mathbf{B}}_{i}=w_{i} \mathbf{Q}^{1 / 2} \mathbf{B}_{i}
$$

where

$$
\mathbf{Q}^{1 / 2} \mathbf{Q}^{-1 / 2}=\mathbf{I}, \quad \mathbf{Q}=\left(\mathbf{Q}^{1 / 2}\right)^{T} \mathbf{Q}^{1 / 2}
$$

By substituting (16) into (15) one obtains a simplified version for upper bound of shakedown limit load (primal problem)

$$
\begin{aligned}
& \alpha^{+}=\min \sum_{k=1}^{m} \sum_{i=1}^{N G}\left(\mu_{i}-\kappa_{i} \sigma_{i}\right) \sqrt{\dot{\mathbf{k}}_{i k}^{T} \dot{\mathbf{k}}_{i k}} \\
\text { s.t.: }\{ & \begin{cases}\sum_{k=1}^{m} \dot{\mathbf{k}}_{i k}-\hat{\mathbf{B}}_{i} \dot{\mathbf{q}}=\mathbf{0} & \forall i=\overline{1, N G} \\
\sum_{i=1}^{N G} \sum_{k=1}^{m} \dot{\mathbf{k}}_{i k}^{T} \mathbf{t}_{i k}-1=0 & \end{cases}
\end{aligned}
$$

It is seen that in the case of limit analysis there exists a dual form for (18), see, e.g. Heitzer and Staat [16], Andersen et al. [17]. Vu et al. [18], [19] generalized this theory for the case of shakedown analysis. An extension of their theory to shakedown analysis of plate is investigated in this work through the following theorem:

Theorem. If there exists a finite solution $\alpha^{+}$for the upper bound shakedown limit load multiplier (20) then the static formulation (12) is exactly the dual problem of the kinematic one (20) such that

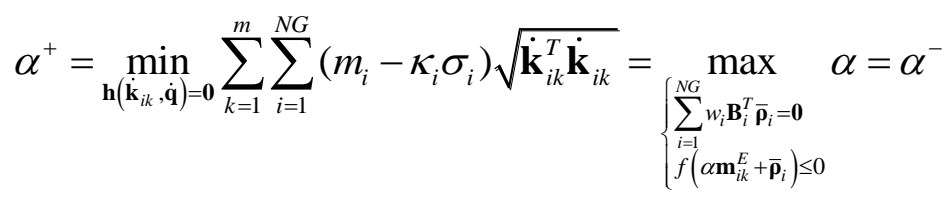

\subsection{Primal-dual shakedown algorithm}

A difficult happening when dealing with the non-linear constrained optimization problem (20) is that the objective function is not everywhere differentiable. In order to allow a direct non-linear, smooth optimization problem, a 'smooth regularization method' should be used for overcoming this barrier. For this purpose, a very small positive number, $\varepsilon_{0}^{2}$ will be added to $D_{\text {int }}\left(\dot{\chi}_{k}\right)$. An efficient technique for large-scale optimization problems, which are successfully applied in [13] is used. Using penalty method to eliminate the first constraint in (18) lead to a penalty function

$$
P=\sum_{i=1}^{N G}\left\{\sum_{k=1}^{M}\left(\left(m_{i}-\kappa_{i} \sigma_{i}\right) \sqrt{\dot{\mathbf{k}}_{i k}^{T} \dot{\mathbf{k}}_{i k}+\varepsilon_{0}^{2}}\right)+\frac{c}{2}\left(\sum_{k=1}^{m} \dot{\mathbf{k}}_{i k}-\hat{\mathbf{B}}_{i} \dot{\mathbf{q}}\right)^{T}\left(\sum_{k=1}^{m} \dot{\mathbf{k}}_{i k}-\hat{\mathbf{B}}_{i} \dot{\mathbf{q}}\right)\right\}
$$

where $c$ is a penalty parameter such that $c \gg 1$. The corresponding Lagrangian of (20) is

$$
L=P-\alpha\left(\sum_{i=1}^{N G} \sum_{k=1}^{m} \dot{\mathbf{k}}_{i k}^{T} \mathbf{t}_{i k}-1\right)
$$


We denote

$$
\boldsymbol{\beta}_{i}=-c\left(\sum_{k=1}^{m} \dot{\mathbf{k}}_{i k}-\hat{\mathbf{B}}_{i} \dot{\mathbf{q}}\right)
$$

By employing Newton method to solve the KKT conditions of the Lagrangian in (39) and after some manipulations, one gets the following system:

$$
\mathbf{K} d \dot{\mathbf{q}}=-\mathbf{K} \dot{\mathbf{q}}+\mathbf{f}_{1}+\mathbf{f}_{2}(\alpha+\mathrm{d} \alpha)
$$

in which

$$
\begin{aligned}
& \mathbf{K}=\sum_{i=1}^{N G} \hat{\mathbf{B}}_{i}^{T} \mathbf{E}_{i}^{-1} \hat{\mathbf{B}}_{i} \\
& \mathbf{f}_{1}=-\sum_{i=1}^{N G} \hat{\mathbf{B}}_{i}^{T} \mathbf{E}_{i}^{-1} \sum_{k=1}^{m} \mathbf{M}_{i k}^{-1}\left(\boldsymbol{\beta}_{i}+\alpha \mathbf{t}_{i k}\right) \frac{\dot{\mathbf{k}}_{i k}^{T} \dot{\mathbf{k}}_{i k}}{\sqrt{\dot{\mathbf{k}}_{i k}^{T} \dot{\mathbf{k}}_{i k}+\varepsilon_{0}^{2}}} \\
& \mathbf{f}_{2}=\sum_{i=1}^{N G} \hat{\mathbf{B}}_{i}^{T} \mathbf{E}_{i}^{-1} \sum_{k}^{m} \mathbf{M}_{i k}^{-1} \sqrt{\dot{\mathbf{k}}_{i k}^{T} \dot{\mathbf{k}}_{i k}+\varepsilon_{0}^{2}} \mathbf{t}_{i k}
\end{aligned}
$$

Solving the system (23), we have the incremental vectors of nodal variables $\dot{\mathbf{q}}$, curvature rate $\dot{\mathbf{k}}_{i k}$ and $\boldsymbol{\beta}_{i}$ as follows :

$$
\begin{aligned}
& d \dot{\mathbf{q}}=d \dot{\mathbf{q}}_{1}+d \dot{\mathbf{q}}_{2}(\alpha+d \alpha) \\
& d \dot{\mathbf{k}}_{i k}=\left(d \dot{\mathbf{k}}_{i k}\right)_{1}+\left(d \dot{\mathbf{k}}_{i k}\right)_{2}(\alpha+d \alpha) \\
& d \boldsymbol{\beta}_{i}=\left(d \boldsymbol{\beta}_{i}\right)_{1}+\left(d \boldsymbol{\beta}_{i}\right)_{2}(\alpha+d \alpha)
\end{aligned}
$$

and

$$
(\alpha+d \alpha)=\frac{1-\sum_{i=1}^{N G} \sum_{k=1}^{m} \mathbf{t}_{i k}^{T}\left[\dot{\mathbf{k}}_{i k}+\left(d \dot{\mathbf{k}}_{i k}\right)_{1}\right]}{\sum_{i=1}^{N G} \sum_{k=1}^{m} \mathbf{t}_{i k}^{T}\left(d \dot{\mathbf{k}}_{i k}\right)_{2}}
$$

The vectors diq $, \mathrm{d} \dot{\mathbf{k}}_{i k}, \mathrm{~d} \boldsymbol{\beta}_{i}$ and $\mathrm{d} \alpha$ are actually Newton directions which assure that a suitable step along them will lead to a decrease of the objective function of the primal problem (18) and to an increase of the objective function of the objective function of the dual problem (12). Based on(25), (26) we can update the vectors of $\dot{\mathbf{q}}, \dot{\mathbf{k}}_{i k}, \boldsymbol{\beta}_{i}$ and $\alpha$.

\section{NUMERICAL EXAMPLES}

In this section, the numerical solution of some problems is presented to test the performance of the dual shakedown algorithm. Plates subjected to uniform or concentrated loads are considered. The 4-node DKQ plate element is applied for structural discretization. For all examples the following was assumed: length $L=10 \mathrm{~m}$, plate thickness $t=0.1 \mathrm{~m}$, the 
mean value of yield stress $\mathrm{E}\left(\sigma_{y}\right)=250 \mathrm{MPa}$ and the standard deviation $\sigma=0.1 \mathrm{E}\left(\sigma_{y}\right)$. A reliability level $\psi=0.9999$ is chosen.
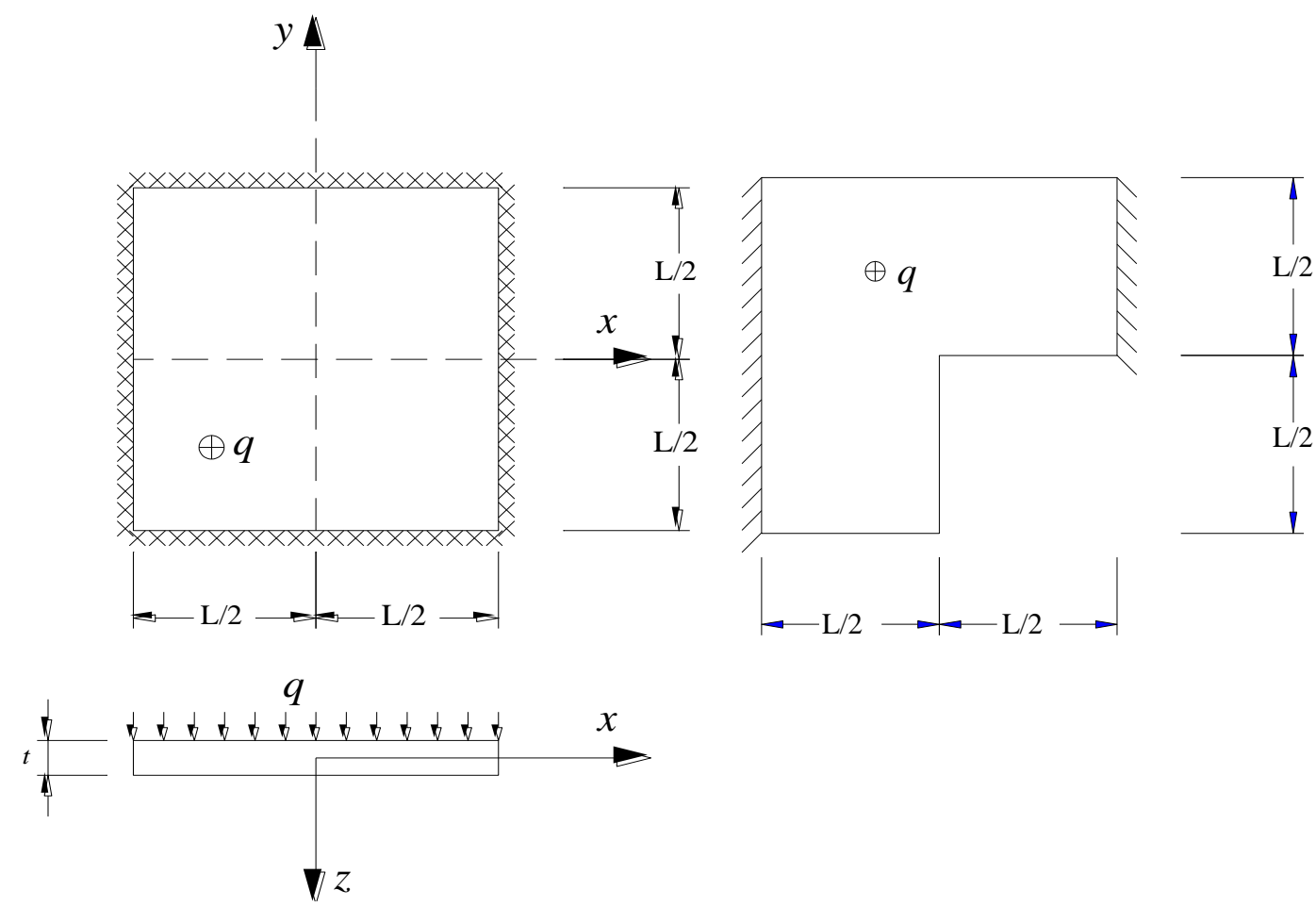

Figure 1. Square plate and L-shape plate loaded by a uniform pressure

\subsection{Limit analysis of square plate subjected to uniform load}

Firstly, we consider a square plate subjected to uniform pressure $q$ as shown on Figure 1. In this analysis, the plate is modelled by 256 DKQ elements due to symmetry. Table 1 shows the comparison of the present numerical results for both cases, simply supported and clamped plates.

\begin{tabular}{lcccc}
\hline \multirow{2}{*}{ Authors } & \multicolumn{2}{c}{ Upper/Lower bound (deterministic) } & \multicolumn{2}{c}{$\begin{array}{c}\text { Upper/Lower bound } \\
\text { (random strength) }\end{array}$} \\
\cline { 2 - 5 } & Spl. supported & Clamped & Spl. supported & Clamped \\
\hline Hodge et al. [14] & $26.54 / 24.86$ & $49.25 / 42.86$ & $-/-$ & $-/-$ \\
Lubliner [17] & $27.71 / 23.81$ & $52.01 /-$ & $-/-$ & $-/-$ \\
Capsoni et al. [15] & $25.02 /-$ & $45.29 /-$ & $-/-$ & $-/-$ \\
Le et al. [16] & $25.01 /-$ & $45.29 /-$ & $-/-$ & $-/-$ \\
Present & $25.04 / 25.04$ & $45.06 / 45.06$ & $15.72 / 15.72$ & $28.36 / 28.36$ \\
\hline
\end{tabular}

Table 1. Limit load factor of square plates in comparison with other solutions $\left(\frac{m_{0}}{q L^{2}}\right)$. 


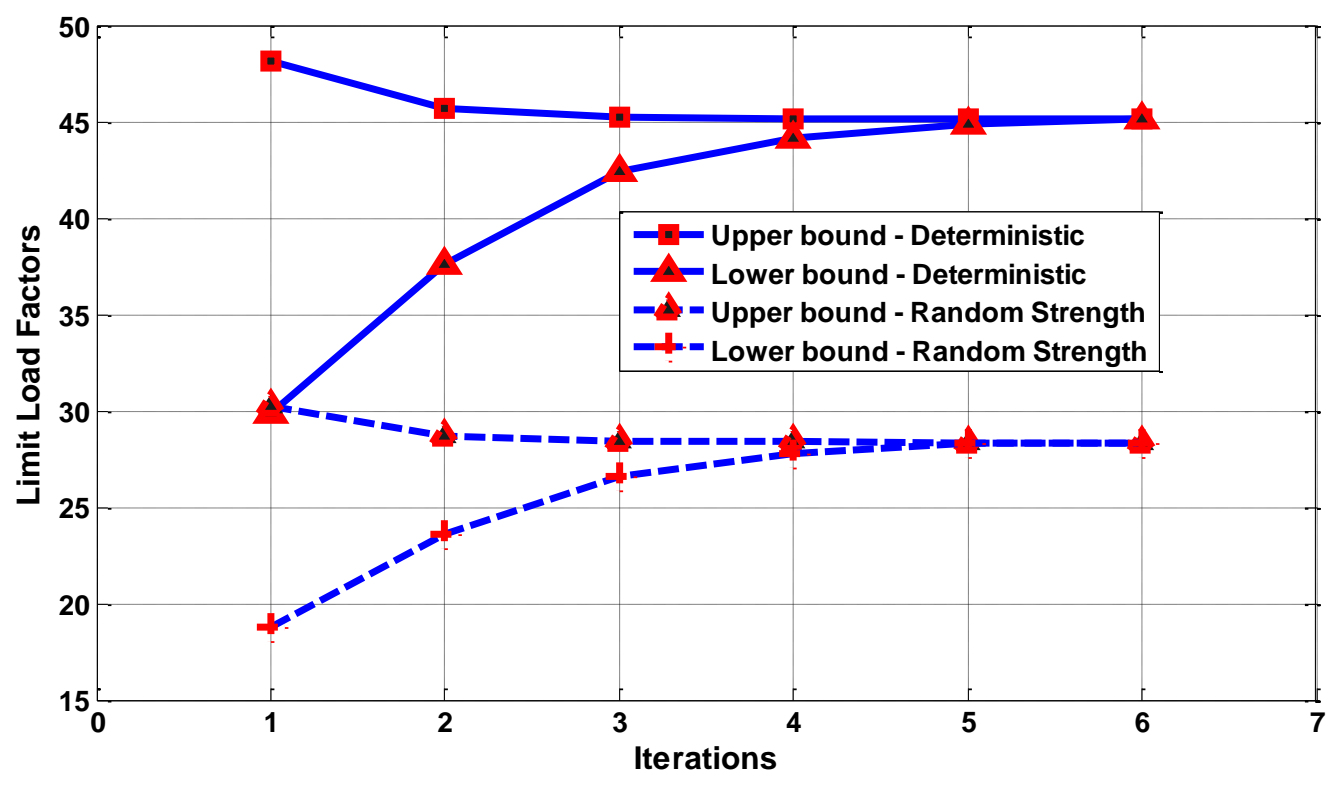

Figure 2. Clamped square plate: Convergence of limit load factors.

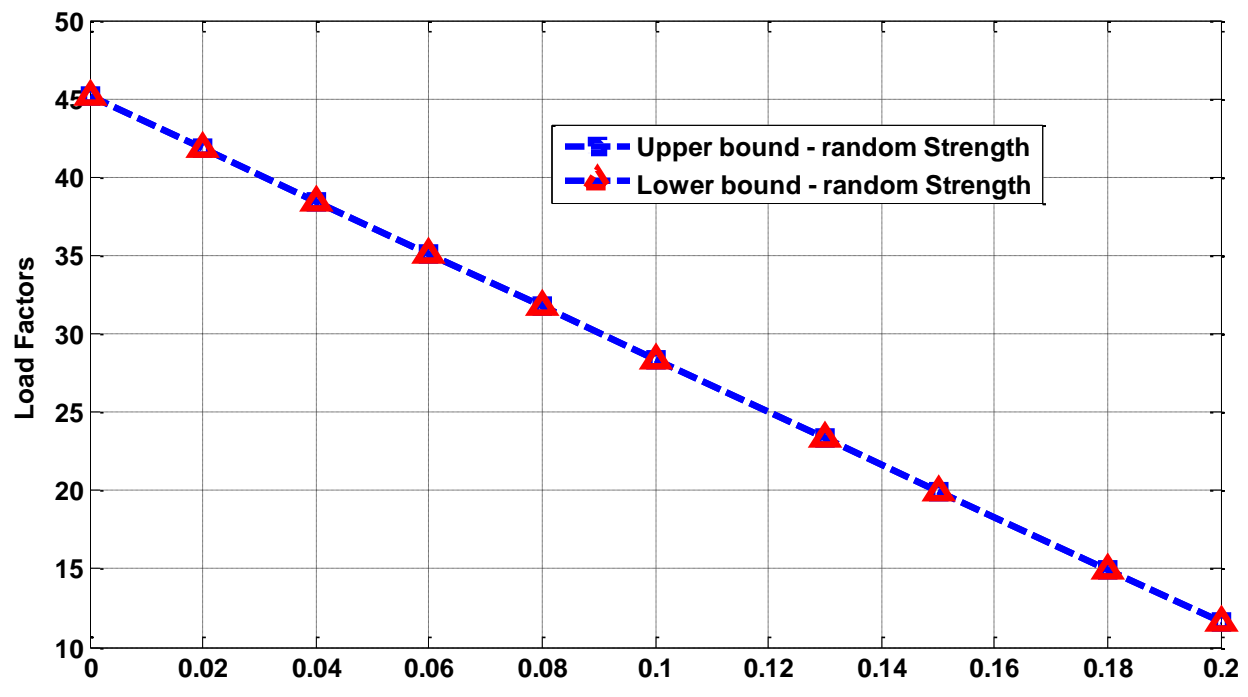

Figure 3. Dependence of load factors on the coefficient of variation $\xi\left(\xi=\frac{\sigma}{E}\right)$.

\subsection{L-shaped plate subjected to uniform load.}

In the second example, we investigate an L-shape plate subjected to uniform pressure $q$ (Figure 1) which can be constant or vary within a range $q \in\left[\begin{array}{ll}0 & q_{\max }\end{array}\right]$. In this analysis, the plate is modelled by 768 DKQ elements. Figure 4 and figure 5 show the convergence of the upper bound and lower bounds for the simply supported case. 


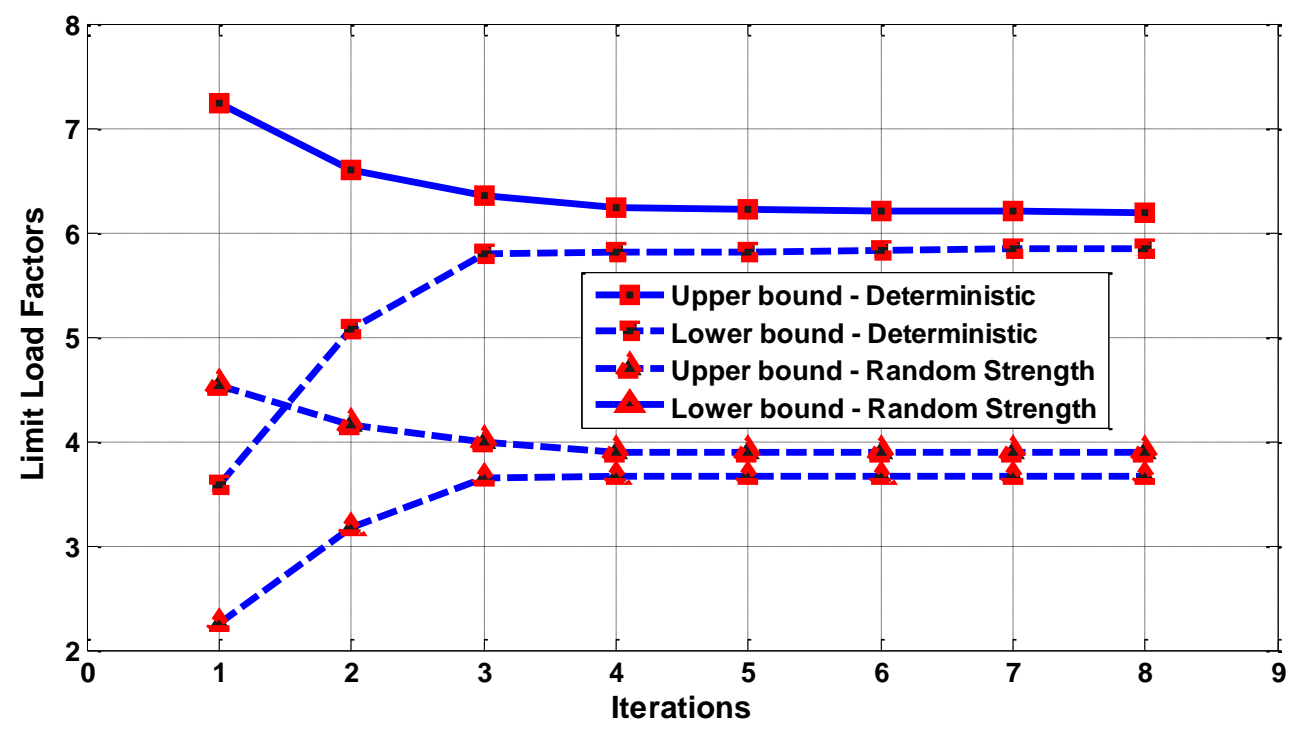

Figure 4: L-shape Plate: Convergence of limit load factors.

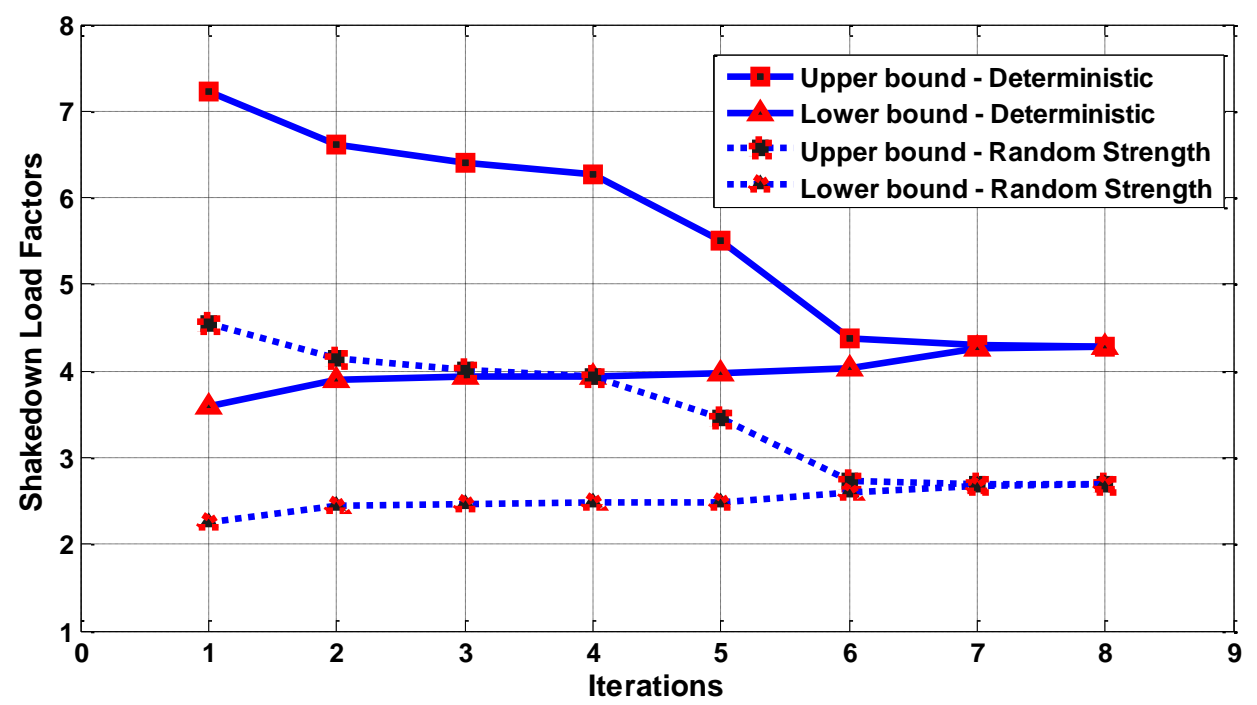

Figure 5: L-shape Plate: Convergence of shakedown load factors

\begin{tabular}{lcccc}
\hline \multirow{2}{*}{ Authors } & \multicolumn{2}{c}{$\begin{array}{c}\text { Upper/Lower bound } \\
\text { (deterministic) }\end{array}$} & \multicolumn{2}{c}{$\begin{array}{c}\text { Upper/Lower bound } \\
\text { (random strength) }\end{array}$} \\
\cline { 2 - 5 } & Limit & Shakedown & Limit & Shakedown \\
\hline Le et al. $[16]$ & $6.289 /-$ & $-/-$ & $-/-$ & $-/-$ \\
Present & $6.19 / 5.85$ & $4.28 / 4.28$ & $3.89 / 3.67$ & $2.69 / 2.69$ \\
\hline
\end{tabular}

Table 2. Limit load factor of plate in comparison with other solutions $\left(\frac{m_{0}}{q L^{2}}\right)$ 


\section{CONCLUSIONS}

- Direct structural reliability design can be achieved by chance constraint programming.

- In the general case chance constraint programming is a hard problem because probabilities have to be calculated as high dimensional integrals during in the optimization algorithm.

- For normally distributed stochastic variables deterministic equivalents can be formulated (for linear programming).

- Extension to nonlinear programming is on its way.

\section{For engineering design:}

- Structural reliability is post design

- Stochastic programming is (pre) design

- The load factor decreases "quickly" with increasing coefficient of variation of the strength.

- High structural reliability can be achieved with moderate reduction of the load factor

\section{REFERENCES}

[1] M. Staat, M Heitzer, Probabilistic limit and shakedown problems. In: M. Staat, M. Heitzer (eds.) Numerical methods for limit and shakedown analysis. Deterministic and probabilistic approach. Part VII. NIC Series Vol. 15, Jülich (2003) 217-268. http://hdl.handle.net/2128/2926

[2] N.T. Trần, T.N. Trần, H.G. Matthies, G.E. Stavroulakis, M. Staat, FEM shakedown analysis of uncertain structures by chance constraint programming. Joint DMV and GAMM Annual Meeting, March 7-11, 2016, Braunschweig, Germany.

[3] T.N. Tran, A dual algorithm for shakedown analysis of plate bending. International Journal for Numerical Methods in Engineering 86, 862-875, 2011.

[4] Shinji Kataoka, A Stochastic programming model, Econometrica, 31, 181-196, 1963.

[5] A. Charnes, W.W. Cooper, Chance-constrained programming. Management Science, 6(1), 73-79, 1959. http://dx.doi.org/10.1287/mnsc.6.1.73

[6] S.S. Rao, Engineering optimization. theory and practice. 4th ed. John Wiley, Hoboken, New Jersey (2009). http://dx.doi.org/10.1002/9780470549124

[7] K.A. Sikorski, A. Borkowski, Ultimate load analysis by stochastic programming. In: D.L. Smith (ed.) Mathematical programming methods in structural plasticity. CISM Courses and Lectures No. 299, Springer, Wien (1990) 403-424. http://dx.doi.org/10.1007/978-3-7091-2618-9_20

[8] F. Tin-Loi, L. Qi, Z. Wei, R.S. Womersley, Stochastic ultimate load analysis: models and solution methods. Numer. Func. Anal. Opt., 17(9-10), 1029-1043, 1996. http://dx.doi.org/10.1080/01630569608816740. 
[9] T.N. Tran, R. Kreißig, M. Staat, Probabilistic limit and shakedown analysis of thin $\begin{array}{lllll}\text { plates and shells. Structural Safety, } & \mathbf{3 1}(1), & 1-18,\end{array}$ http://dx.doi.org/10.1016/j.strusafe.2007.10.003

[10] T.N. Tran, M. Staat, Uncertain multimode failure and shakedown analysis of shells. In: P. Fuschi, A.A. Pisano, D. Weichert (eds.) Direct methods for limit and shakedown analysis of structures. Chap. 14. Springer, (2015) 279-298. http://dx.doi.org/10.1007/978-3-319-12928-0_14

[11] D.K. Vu, A.M. Yan, D.H. Nguyen, A primal-dual algorithm for shakedown analysis of structure. Computer methods in Applied Mechanics and Engineering 193, 4663-4674, 2004

[12] P.G. Hodge, T. Belytschko, Numerical methods for the limit analysis of plates. Transactions of ASME, Journal of Applied Mechanics 35, 796-801, 1986.

[13] A. Capsoni, L. Corradi, Limit analysis of plates - a finite element formulation. Structural Engineering and Mechanics 8, 325-341, 1999.

[14] V.C. Le, M. Gilbert, H. Asker, Limit analysis of plates using the EFG method and second-order cone programming. International Journal for Numerical Methods in Engineering 78, 1532-1552, 2009.

[15] J. Lubliner, Plasticity theory. Macmillan, 1990.

[16] M. Heitzer, M. Staat: FEM-computation of load carrying capacity of highly loaded passive components by direct methods. Nuclear Engineering and Design 193, 349-358, 1999. http://dx.doi.org/10.1016/S0029-5493(99)00190-9

[17] K.D. Andersen, E. Christiansen, A.R. Conn, M.L. Overton, An efficient primal-dual interior-point method for minimizing a sum of Euclidean norms. SIAM Journal on Scientific Computing 22, 243-262, 2000.

[18] D.K. Vu, A.M. Yan, D.H. Nguyen, A dual form for discretized kinematic formulation in shakedown analysis. International Journal of Solid and Structures 41, 267-277, 2004.

[19] D.K. Vu, M, Staat, Shakedown analysis of structures made of materials with temperature-dependent yield stress. International Journal of Solids and Structures, 44(13), 4524-4540, 2007. http://dx.doi.org/10.1016/j.ijsolstr.2006.11.038

[20] M. Staat, Limit and shakedown analysis under uncertainty. Int. J. Comput. Methods, 11(2), 1343008, 2014. http://dx.doi.org/10.1142/S0219876213430081 